\title{
AVALIAÇÃo de ALTERNATIVAS DE COLHEITA SOB PERSPECTIVA DE COGERAÇÃO ATRAVÉS DE PROGRAMAÇÃO LINEAR INTEIRA MISTA
}

\author{
Felipe Kesrouani Lemos ${ }^{1,3}$, Camila Furlan Moraes ${ }^{2}$, Marcelo Pondé do Vale ${ }^{2}$ \\ ${ }^{1}$ Docente, ${ }^{2}$ Discente do Curso de Engenharia de Produção da UNOESTE, Presidente Prudente, SP; ${ }^{3}$ Pós-Graduando em Engenharia \\ de Produção na Escola Politécnica da USP, São Paulo, SP. e-mail: felipeklemos@unoeste.br
}

\section{RESUMO}

O setor sucroalcooleiro não só é de grande importância econômica para o país, como também tem sido o centro de novas possibilidades energéticas renováveis. Além do próprio etanol, a cogeração é um advento recente da mudança de regra na produção e comercialização de eletricidade no país. O objetivo deste estudo é propor um modelo de planejamento agregado de produção - em formulação matemática linear inteira mista - considerando os produtos (álcool anidro e hidratado) e subprodutos (energia e bagaço in natura) de uma indústria sucroalcooleira. Para isso, são abarcadas não só decisões de produção, venda e estocagem, como também de métodos alternativos de colheita com maiores ou menores graus de palha, insumo este que pode ser aproveitado na cogeração, da mesma forma que o bagaço in natura de cana-deaçúcar. Os resultados testados com dados de uma usina do interior de São Paulo mostram que pode existir interesse em colheitas menos eficientes na limpeza da palha da cana para aumentar o resultado global através dos subprodutos. Os resultados preliminares levaram a um interesse em refinamento dos parâmetros, sendo destacado como produto da pesquisa o instrumento de apoio à decisão elaborado. Futuros desdobramentos incluem a consideração de outras variáveis de processos que são alteradas pela proporção de palha na mistura.

Palavras-chave: planejamento de produção, programação linear inteira mista, indústria sucroalcooleira, cogeração, otimização.

\section{EVALUATION HARVEST ALTERNATIVES UNDER COGENERATION PERSPECTIVE THROUGH MIXED INTEGER LINEAR PROGRAMMING}

\begin{abstract}
The sugar and alcohol sector has great relevance to Brazil's economy and, also, has been the center of new renewable energy possibilities. Besides ethanol itself, cogeneration is a recent advance which changed rules on production and sales of electricity in the country. The objective of this paper is to propose a master production planning model - with mixed linear integer mathematical formulation - considering products and byproducts of a alcohol and sugar factory. Not only production, sales and stocks decision are considered, but also alternatives of harvest methods with different efficiencies of straw, which can be used to cogenerate energy. Results were tested with data of a factory of western São Paulo, showing that it might be interesting to use less efficient methods of harvest to increase global results through byproducts. Preliminary results brought interest of refining data and parameters, having special attention to the decision support tool created. Future works include considering other process variables affected with straw portion on harvest.

Keywords: production planning, mixed integer linear programming, sugar and alcohol industry, cogeneration; optimization.
\end{abstract}




\section{INTRODUÇÃO}

Desde a reforma do setor elétrico brasileiro, em que o setor privado pôde participar de investimentos e operações na produção de energia, a cogeração passou a ser uma opção viável tecnicamente para a indústria, em particular, para 0 setor sucroalcooleiro (NAGAOKA et al., 2003). Concomitantemente, existe uma pressão e estímulo para o aumento da participação de fontes renováveis na matriz energética, o que torna a produção de energia por biomassa ainda mais atrativa.

Desta forma, as usinas de álcool e açúcar passam a ter um trade-off na produção de subprodutos, já que o bagaço de cana pode ser transformado tanto em energia elétrica, quanto em bagaço in natura para uso em diversas aplicações, como, por exemplo, confinamentos de bovinos de corte. Além disto, a separação da palha na cana-de-açúcar colhida passa a ser posta em questionamento, já que esta pode ser matéria-prima para a produção de energia.

O objetivo deste trabalho é desenvolver um modelo de programação linear inteira mista para o planejamento de produção neste setor, permitindo analisar o mix de produção, levando em conta o trade-off na produção de seus produtos e subprodutos; e os custos incorridos em diferentes métodos de colheita (com menores ou maiores graus de palha). Assim, tem-se como escopo as decisões de quantidades a serem produzidas e as formas de colheita.

Recorrendo à tipologia de metodologias quantitativas em modelagem e simulação de Morábito e Pureza (2010), pode-se classificar esta pesquisa como empírica prescritiva, já que apresenta como intuito a adesão entre a realidade deste sistema de produção e o modelo proposto; e ainda, busca apoiar o processo de decisões associadas ao planejamento de produção neste setor.
Este trabalho está organizado da seguinte forma: apresenta introdução que traça os objetivos gerais do trabalho, posicionando também o setor estudado e o escopo de modelagem; uma revisão bibliográfica com são levantados os trabalhos de modelagem no setor sucroalcooleiro; a Formulação Matemática do modelo, em que são apresentados os parâmetros, variáveis e o equacionamento destes formando função objetivo e restrições; Experimentos, em que é relatada a coleta de dados feita para a utilização do modelo em um caso de uma usina sucroalcooleira do interior do estado de São Paulo, bem como os resultados obtidos. Posteriormente, é feita uma análise destes resultados e uma conclusão do trabalho.

\section{REVISÃO DA LITERATURA}

Devido à extensão do território brasileiro, ao favorecimento do clima para a produção agroindustrial no país e à variedade de produtos da cana-de-açúcar, são cada vez mais freqüentes estudos voltados para o setor sucroalcooleiro.

Miranda (2009) afirma que em países como o Brasil, que possui inúmeras áreas de terra fértil, insolação abundante e possibilidade de recursos hídricos, o cultivo da cana-de-açúcar para posterior utilização dos seus resíduos de seu processamento (bagaço de cana-de-açúcar) e colheita (palha da cana-de-açúcar) com o intuito de geração de eletricidade é uma alternativa viável para a produção de energia elétrica para suprir as necessidades do mercado. Mesquita (2008) mencionou a importância que se fazer uma abordagem do Sistema Interligado Nacional sob o ponto de vista do impacto da inserção de potência pelas usinas termelétricas a biomassa de cana-de-açúcar, onde deverão ser realizados estudos de aproveitamento energético nas plantas industriais sucroalcooleiras, visando um aumento do potencial de exportação de energia. 
A otimização do consumo de vapor, a substituição de motores convencionais por alto rendimento, uso de combustíveis alternativos possibilitando a geração de energia durante todo 0 ano, são alguns dos aspectos que precisam ser melhor explorados. Santos (2010) aborda a tendência dos bicombustíveis que, hoje, surgem como uma nova opção na matriz energética mundial, principalmente pelas novas políticas sustentáveis, que buscam alternativas de energias renováveis de baixo custo.

Em termos da aplicação de técnicas de otimização no setor, lanonni e Morábito (2002) utilizam-se de simulação discreta para analisar a operação logística da balança de recepção até as moendas, comparando decisões de diferentes modais e tipos de cana (picada ou inteira). Colin (2009) propõe um modelo para o tempo de replantio, chegando a conclusão que há ganhos de produtividade o ciclo de 4 anos em relação ao mais utilizado ciclo de 6 anos. Milan et al. (2006) abordam a questão logística da colheita até a usina no contexto cubano através de programação linear inteira mista. Já Calija et al. (2001) utilizam simulação estocástica e programação dinâmica para modelar o processo de seleção de variedades e clones de cana-deaçúcar.

No contexto de planejamento, Paiva e Morábito (2007) apresentam apresentou um modelo de otimização para o planejamento agregado da produção de usinas de açúcar e álcool baseando-se nos modelos clássicos de seleção de processos e dimensionamento de lotes para representar o sistema de produção de açúcar, álcool e melaço, incluindo decisões da etapa agrícola, das fases de corte, carregamento e transporte de cana e, principalmente, decisões de moagem, escolha do processo produtivo e estoque dos produtos finais. Kostin et al. (2012) abordam a questão da infraestrutura sob a ótima do planejamento estratégico, no contexto
Argentino, tendo como variáveis as expansões de produção de instalações de estocagem, contemplando a questão de mitigação de risco sob diferentes cenários.

Bezuidenhout e Baier (2011) fazem a consideração de que existe uma abundância de modelos matemáticos em relação a modelos e longo prazo, porém uma escassez nos de curto prazo.

\section{FORMULAÇÃO MATEMÁTICA}

Seja uma usina do setor sucroalcooleiro que $\mathrm{J}$ produtos $(\mathrm{j}=1, \ldots, \mathrm{J})$, sendo que cada um possui um preço de venda $p_{j}$. Sejam I as matérias-primas $(i=1, \ldots I)$ para a produção de cada um destes produtos, sendo que $\theta_{i j j}$ é a quantidade de matéria-prima i necessária para a produção de uma unidade do produto j. Sejam $\mathrm{K}$ as formas de colheita $(\mathrm{K}=1, \ldots, \mathrm{K})$, que podem variar de acordo com a forma com que a palha é trazida para a indústria. Cada forma de colheita $\mathrm{k}$ produz uma quantidade da matéria-prima i $m_{i k}$ por hectare. de acordo com o nos canaviais, sendo que possui também um custo de colheita, $C C_{k k f}$, que depende de $k$ e também da fazenda $f(f$ $=1, \ldots, F)$, com área $A_{f}$, em que é colhida.

Deve-se salientar também que alguns dos produtos, como o álcool anidro e a energia possuem contratos de fornecimento atrelados, sendo a quantidade mínima necessária a ser fornecida em um mês $t$ denotada por $C_{j \mathrm{jt}}$. A capacidade máxima de estocagem de um produto j é dada por ${ }^{C A P_{j}^{P A}}$, enquanto a capacidade de produção de um um produto j no mês té dada por CAP Prod $^{\text {Prod }}$. O uso da palha cada a geração de energia leva a um custo fixo de $\varpi$.

Como variáveis do modelo, temos $x_{j t}$, a quantidade a ser produzida do produto $j$ no período $t ; s_{j t}$, que é a quantidade do produto $j$ que é vendida no período $\mathrm{t}{ }^{S T K_{j t}^{P A}}$, que é a 
quantidade estocada do produto j no período t. Além disto, $Y_{k f t}$ é uma variável binária que vale 1 se a fazenda f é colhida pelo método $\mathrm{k}$ no mês $\mathrm{t}$, e 0 caso contrário. Por fim, $\varphi$ é um binário que vale 1 se é gerada energia a partir de palha e 0 caso contrário.

O modelo (1)-(9) retrata o objetivo e as restrições do sistema. Como objetivo (1) temos a diferença entre a somatória das receitas com cada um dos produtos e os custos de colheita com cada fazenda no método escolhido. Também é descontado o custo fixo de processamento da palha. Fica claro aqui que o modelo adota uma simplificação que os custos variáveis dos produtos são insignificantes frente aos custos fixos do processo.

A restrição (2) faz um balanço entre a quantidade consumida de uma matéria-prima i em um certo mês $\mathrm{t}$ e a a sua produção através das colheitas nos diferentes métodos, nas diferentes fazendas. A restrição (3) impõe que somente um mês é usado por fazenda e cada fazenda também só tem colheita em um único mês. A restrição (4) impõe uma venda limitada inferiormente pelos contratos assumidos pela empresa. As restrições (5) retratam o balanço de estoques: a quantidade em estoque de um determinado produto j em um período t é a quantidade no período anterior mais a produção, menos as vendas. Em (6) essa quantidade estocada à capacidade de estoque da empresa. Em (7) é a quantidade produzida que é limitada superiormente pela capacidade de produção da empresa. Finalmente, em (8), dá-se sentido à variável $\varphi$, fazendo-a coerente com seu signicado físico (sendo $U B_{\text {energia }}$ um limitante superior da quantidade de energia produzida com palha.

$$
\begin{aligned}
& \max \sum_{j=1}^{J} \sum_{t=1}^{T} p_{j} S_{j t}-\sum_{i=1}^{T} \sum_{t=1}^{T} \sum_{f=1}^{F} C C_{k f f} y_{k f t}-\varphi w \\
& \text { sa } \sum_{j=1}^{J} \theta_{i j} x_{j t} \leqslant \sum_{k=1}^{K} \sum_{f=1}^{F} A_{f} m_{i k} Y_{k f t} \quad i=1_{s, n} I, \quad t=1, \ldots, T \\
& \sum_{k=1}^{K} \sum_{t=1}^{T} y_{k f t}=1 \quad f=1_{s m s} F \\
& s_{j t} \geq C_{j t} \quad j=1_{s \omega x} J, t=1_{s m s} T \\
& 5 T K_{j t}^{P A}=5 T K_{j t-1}^{P A}+x_{j t}-s_{j t} \quad j=1_{s m o} j_{0} \quad t=1_{s m o} T
\end{aligned}
$$

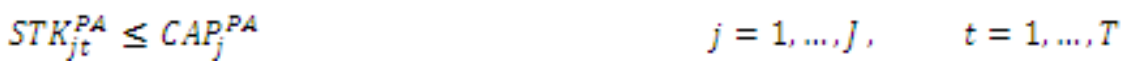

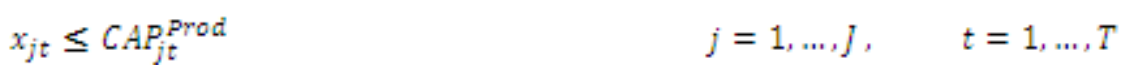

$$
\begin{aligned}
& s_{j t} \leq U B_{\text {energia } \varphi} \quad \forall j, \theta_{i j} \geq 0, \operatorname{com} i \text { sendo palh } a_{s} \quad t=1_{s, n} T
\end{aligned}
$$




$$
s_{j t}, x_{j t}, S T K_{j t}^{P A} \in \mathbf{R}_{+} \quad, \quad \varphi, y_{k f t} \in\{0,1\}
$$

\section{EXPERIMENTOS E RESULTADOS COMPUTACIONAIS}

\section{Coleta de dados}

Este modelo foi rodado utilizando os dados coletados em uma usina sucroalcooleira do interior de SP. Os dados foram obtidos através do gerente de cogeração da empresa, com auxílio de outras áreas.

A Tabela 1 mostra os produtos $\mathrm{e}$ subprodutos (j) produzidos pela empresa, com seus respectivos preços $\left(\mathbb{P}_{\mathfrak{j}}\right)$. Ressalta-se aqui que os preços foram considerados constantes ao longo do ano, o que é uma premissa simplificadora, dado que existem variações.
Como não existe uma estratégia de preços em termos de previsão de suas oscilações, estes foram considerados estáticos ao longo do ano por questões de simplificação. Também são mostrados na tabela os contratos mínimos de fornecimento $\left(C_{j \mathrm{j}}\right)$, ressaltando que aqui são mostrados como constantes em t, porém a energia possui contrato nulo quando t não é mês de colheita. Da mesma forma, as capacidades de produção ( ${ }^{C A P_{j t}^{\text {Prod }}}$ ) são mostradas, porém são reduzidas a zero em meses $\mathrm{t}$ em que não há colheita (dezembro, janeiro, fevereiro e março). Por fim, também é mostrada a capacidade de estocagem ( $\left.{ }^{C A P_{j}^{P A}}\right)$ de cada produto.

Tabela 1. Produtos e subprodutos produzidos pela empresa e seus parâmetros relacionados.

\begin{tabular}{|c|c|c|c|c|}
\hline Produtos (j) & $\begin{array}{c}\text { Preço } \\
\left(\mathbb{P}_{j}\right)\end{array}$ & $\begin{array}{l}\text { Contrato } \\
\left(C_{j t}\right)\end{array}$ & $\begin{array}{l}\text { Capacidade de } \\
\text { estocagem } \\
\left(C A P^{P A}\right)\end{array}$ & $\begin{array}{c}\text { Produção } \\
\text { máxima }\left({ }^{C A P_{j t}^{\text {Prod }}}\right)\end{array}$ \\
\hline Álcool hidratado (L) & 1,30 & 0 & $60,000,000$ & $28,800,000$ \\
\hline Álcool anidro (L) & 1,51 & $4,500,000$ & $20,000,000$ & $6,750,000$ \\
\hline Bagaço $(t)$ & $R \$ \quad 50,00$ & 0 & $\infty$ & $\infty$ \\
\hline $\begin{array}{l}\text { Energia de bagaço (MWh) } \\
\text { Energia de palha (MWh) }\end{array}$ & $\begin{array}{l}\mathrm{R} \$ 200,00 \\
\mathrm{R} \$ 200,00\end{array}$ & 21,600 & 0 & 43,200 \\
\hline
\end{tabular}

As matérias-primas i para o processo de produção destes produtos são três: caldo, bagaço e palha. A Tabela 2 exibe as quantidades (em $\mathrm{kg}$ ) necessárias de cada matéria-prima para a produção de 1 unidade de cada produto $\mathrm{j}$.
Tabela 2. Quantidades de cada matéria-prima i para cada produto $\mathrm{j}\left(\theta_{\mathrm{i} j \tilde{j}}\right)$.

\begin{tabular}{lccc}
\hline \multirow{2}{*}{ Produtos $(\mathrm{j})$} & \multicolumn{3}{c}{ MPs $\left(\boldsymbol{\theta}_{i j}\right)$} \\
\cline { 2 - 4 } & $\begin{array}{c}\text { Caldo } \\
(\mathrm{kg})\end{array}$ & $\begin{array}{c}\text { Bagaço } \\
(\mathrm{kg})\end{array}$ & $\begin{array}{c}\text { Palha } \\
(\mathrm{kg})\end{array}$ \\
\hline Álcool hidratado $(\mathrm{L})$ & 1,9125 & 0 & 0 \\
Álcool anidro $(\mathrm{L})$ & 2,04 & 0 & 0 \\
Bagaço $(\mathrm{t})$ & 0 & 1000 & 0 \\
Energia de bagaço $(\mathrm{MWh})$ & 0 & 1176.5 & 0 \\
Energia de palha $(\mathrm{MWh})$ & 0 & 0 & 1176,5 \\
\hline
\end{tabular}

Os métodos $k$ de colheita foram colocados aqui utilizando a experiência do setor agrícola e fontes técnicas. Atualmente é feito apenas um método e colheita como paradigma: 0 
método 1 , que obtém $8 \%$ do volume transportado sob a forma de palha. É possível ajustar diferentes tipos de limpeza da palha no campo, conseguindo graus de pureza menores. Sem a cogeração, não há interesse nesta palha, buscando-se a maior eficiência na limpeza. Entretanto, com o advento desta, uma análise pode ser procedida. Assim, os métodos de 1 a 4 são diferentes graus de limpeza de palha (veja que os níveis de garapa e bagaço são constantes). Finalmente o método 5 propõe-se a manter o nível de pureza da palha do método 1 (8\%) e abrir uma segunda frente de colheita apenas de palha. A Tabela 3 retrata a quantidade produzida de cada matéria-prima i por tonelada de cana produzida ( $\left.m_{i k}\right)$. Importante ressaltar que a quantidade não totaliza $100 \%$ já que estes são os valores secos (sem água) dos produtos, além de haver transporte de impurezas.

Tabela 3. Quantidades de cada matéria-prima i em cada método de colheita $\mathrm{k}\left(\mathrm{m}_{\text {ik }}\right.$ ).

\begin{tabular}{cccc}
\hline \multirow{2}{*}{$\begin{array}{c}\text { Método } \\
(\mathbf{k})\end{array}$} & \multicolumn{3}{c}{ Quantidades produzidas $\left(\boldsymbol{m}_{i k}\right)$} \\
\cline { 2 - 4 } & Caldo $(\mathrm{kg} / \mathrm{t})$ & Bagaço $(\mathrm{kg} / \mathrm{t})$ & Palha $(\mathrm{kg} / \mathrm{t})$ \\
\hline 1 & 153 & 276 & 80 \\
2 & 153 & 276 & 100 \\
3 & 153 & 276 & 120 \\
4 & 153 & 276 & 140 \\
5 & 153 & 276 & 165 \\
\hline
\end{tabular}

Finalmente, o custo de colheita $\left(C C_{k f f}\right)$ com o método $k$ na fazenda $f$ foi estimado da seguinte forma: o custo de maquinário para a colheita foi estimado pelo setor de custos como $\mathrm{R} \$ 300,00$ por hectare, sendo $\mathrm{R} \$ 60,00$ para cada um dos 5 equipamentos utilizados ( 2 tratores com implemento de corte, 1 pá carregadeira e 2 caminhões). Para o método 5 , este custo é duplicado, já que 2 frentes são abertas para a mesma área. Já o custo de transporte, foi dimensionado - no método 1 - como sendo $R \$$ 1,90 por km (considerando ida e volta), sendo que cada viagem transporta 30 toneladas de cana (1 carreta), ou seja, a produção de 0.5 hectare. Os demais métodos foram considerados proporcionais em relação à perda de capacidade no transporte, ou seja, o aumento da quantidade de palha foi refletido no aumento do gasto com transporte proporcionalmente. As áreas $\mathrm{e}$ distâncias até cada fazenda foram omitidas por uma questão de confidencialidade e extensão dos dados (sem relevância aqui).
Adicionalmente, o custo fixo para utilizar produtos derivados da palha (no caso, a energia da palha) é de aproximadamente $R \$$ $1.000 .000,00$.

\section{RESULTADOS}

O modelo proposto foi implementado em CPLEX 12.3 com interface excel e rodado em um computador com processador i7 $2^{\mathrm{a}}$ geração, com memória RAM $8 \mathrm{~Gb}$. $\mathrm{O}$ tempo de processamento foi de 26 segundos, o que pode ser considerado irrelevante para um instrumento de planejamento de médio prazo.

Como pode ser visto na Tabela 4, o modelo indica que todos os produtos propostos são vendidos. $\mathrm{O}$ álcool anidro, apesar do maior consumo de caldo para sua produção, apresenta-se vantajoso pelo preço, sendo que é produzido até o limite de produção imposto pela cooperativa que compra o produto (sendo, na verdade, um limite de demanda). Quanto aos subprodutos, nota-se também uma preferência, para esta estrutura de 
preços, da energia em relação ao bagaço in com o excedente das caldeiras (atingem a natura, já que este último passa a ser produzido restrição de produção máxima).

Tabela 4. Venda total no ano de cada produto.

\begin{tabular}{lc}
\hline \multicolumn{1}{c}{ Produtos } & Venda total \\
\hline Álcool hidratado $(\mathrm{L})$ & $104,909,472$ \\
Álcool anidro $(\mathrm{L})$ & $54,000,000$ \\
Bagaço $(\mathrm{t})$ & 438,451 \\
Energia de bagaço (MWh) & 103,873 \\
Energia de palha (MWh) & 241,727 \\
\hline
\end{tabular}

A Tabela 5 mostra as áreas de colheita e as distâncias percorridas para cada mês de colheita. Como não há considerações de capacidade, verifica-se um desbalanço nas distâncias (o que não ocorre nas áreas por uma questão de produção e estocagem de matérias- primas). O resultado mais interessante é que em todas as fazendas a solução ótima estava com o método 4 (mínima limpeza da palha na colheita).

Tabela 5. Áreas de colheita e distâncias percorridas por mês no método 4.

\begin{tabular}{ccc}
\hline Mês $(\mathrm{t})$ & Área total (ha) & Distância total $(\mathrm{Km})$ \\
\hline 4 & 4250.04 & 884 \\
5 & 4069.81 & 600 \\
6 & 4219.76 & 3038 \\
7 & 4529.12 & 724 \\
8 & 3601.5 & 241 \\
9 & 3638.47 & 666 \\
10 & 4682.91 & 230 \\
11 & 4864.53 & 681 \\
\hline
\end{tabular}

\section{DISCUSSÃO}

O modelo apresentado mostrou-se interessante para o planejamento agregado da produção em uma indústria sucroalcooleira. Através deste é possível encontrar quantidades a serem produzidas e vendidas mês-a-mês levando em conta restrições de capacidade produtiva, de estocagem, contratos e preços de mercado.

Os resultados mostraram que para os preços colocados existe uma preferência pelo álcool anidro (limitada pela demanda) e pela energia como subproduto, em relação ao bagaço in natura (limitada pela capacidade das caldeiras). Outro resultado interessante é a opção pelo método 4 , em que as máquinas fazem uma limpeza da palha na colheita menor, arcando com os maiores custos de frete, porém com maior disponibilidade deste para a co-geração, mantendo o mercado de bagaço in natura simultaneamente (mesmo com um custo fixo incorrido).

Mais do que os resultados, o método mostrou-se válido na tomada de decisão de planejamento, na medida em que permite analisar diferentes conjunturas de preços para verificar o plano de produção e o fechamento de contratos de energia, para não ficar na dependência dos preços de mercado spot de energia. 
O tempo computacional do modelo é bastante pequeno se considerado que se trata de uma ferramenta com horizonte de médio prazo. Isso sugere que o modelo é computacionalmente viável mesmo para instâncias reais de maior porte.

Os resultados obtidos são preliminares, uma vez que pretende-se fazer uma nova rodada de validações com o planejamento de safra da empresa, possibilitando refinar premissas e melhorar estimativas de parâmetros.

\section{CONCLUSÃO}

O objetivo deste trabalho foi modelar um sistema de produção sucroalcooleiro, sob a ótica da maximização de sua margem entre as receitas com seus produtos e os custos de colheita. Foi feita uma formulação linear inteira mista par ao problema, que considerou diferentes tipos de colheita possíveis e objetivou um mix de produção que maximizasse a margem mencionada.

Este estudo preliminar possibilitou o desenvolvimento do método, que mostra-se útil no apoio a decisões tática.

Como sugestões de pesquisas futuras, estão o desenvolvimento de análises de sensibilidade em relação aos preços do produto e capacidades de produção e estocagem (para melhorar a visão sobre investimentos), considerações de flutuações de preços ao longo dos meses e previsões de demanda.

\section{REFERÊNCIAS}

BEZUIDENHOUT, C. N. \& BAIER, T. J. A. An evaluation of the literature on integrated sugarcane production systems: A scientometrical approach. Outlook on agriculture, Vol. 40, n. 1, p. 79-88, 2011. http://dx.doi.org/10.5367/oa.2011.0025

CALIJA, V.; HIGGINS, A. J.; JACKSON, P. A.; BIELIG, L. M. \& COOMANS, D. An operations research approach to the problem of the sugar cane selection. Annals of Operations Research, Vol. 108, n. 1, p. 123-142, 2001. http://dx.doi.org/10.1023/A:1016054911470

COLIN EMERSON, C. Mathematical programming accelerates implementation of agroindustrial sugarcane complex. European Journal of Operational Research, Vol. 199, n. 1, p. 232235,

2009.

http://dx.doi.org/10.1016/i.ejor.2008.11.016

IANNONI, A. P. \& MORÁBITO, R. Análise do sistema logístico de recepção de cana-de-açúcar: um estudo de caso utilizando simulação discreta. Revista Gestão \& Produção, Vol. 9, n. 2, p. 107128, 2002.

KOSTIN, A. M; GUILLEN-GOSALBEZ, G.; MELE, $F$. D. Design and planning of infrastructures for bioethanol and sugar production under demand uncertainty. Chemical Engineering Research \& Design, Vol. 90, n. 3A, p. 359-376, 2012. http://dx.doi.org/10.1016/j.cherd.2011.07.013

MILAN, E. L.; FERNANDEZ, S. M. \& Aragones, L. M. P. Sugar cane transportation in Cuba, a case study. European Journal of Operational Research, Vol. 174, n. 1, p. 374-386, 2006. http://dx.doi.org/10.1016/j.ejor.2005.01.028

MORÁBITO, R. \& PUREZA, V. Modelagem e simulação, em: Metodologia de Pesquisa em Engenharia de Produção e Gestão de Operações, Paulo A. C. Miguel (ed), Editora Campus/Elsevier, Rio de Janeiro, 165-194, 2010.

NAGAOKA, M. P. T.; Esperancini, M. S. T. \& Pinheiro, F. A. Análise da comercialização de energia elétrica cogerada pelo setor sucroalcooleiro no estado de São Paulo. Revista de Economia e Sociologia Rural, Vol. 41, n. 4, p. 759-778, 2003. http://dx.doi.org/10.1590/S0103$\underline{20032003000400003}$

PAIVA, R. P. O. \& MORÁBITO, R. Um modelo de otimização para o planejamento agregado da produção em usinas de açúcar e álcool. Gestão \& Produção, Vol. 14, n. 1, p. 25-41, 2011. http://dx.doi.org/10.1590/S0104530X2007000100004 